\title{
Once-weekly Dulaglutide and Major Cardiovascular Events-Results of the REWIND Trial
}

\author{
John Doupis
}

Diabetes Division and Clinical Research Center, latriko Paleou Falirou Medical Center, Paleo Faliro, Greece; Internal Medicine, Diabetes Department, NS Naval Hospital, Athens, Greece

$\mathrm{T}$ he REWIND study investigated the effect of the glucagon-like peptide 1 (GLP-1) receptor agonist dulaglutide on major adverse cardiovascular events (MACE) in individuals with type 2 diabetes (T2D) with and without previous cardiovascular disease and with a wide range of glycemic control. At a median follow-up of 5.4 years, the longest to date for a cardiovascular outcome trial for a GLP-1 receptor agonist, the first occurrence of MACE (including non-fatal myocardial infarction, non-fatal stroke, or death from cardiovascular causes) occurred in $12.0 \%$ of dulaglutide-treated patients compared with $13.4 \%$ in the placebo group. Dulaglutide is therefore a useful option in the management of glycemic control in middle-aged and older people with T2D with either previous cardiovascular disease or cardiovascular risk factors.

\section{Keywords}

Cardiovascular outcome trial, dulaglutide, glucagon-like peptide 1 (GLP-1) agonist, type 2 diabetes

Disclosures: John Doupis is a clinical researcher, lecturer and on the advisory board for Eli Lilly, Novo Nordisk and AstraZeneca.

Acknowledgements: Medical writing assistance was provided by Katrina Mountfort from Touch Medical Media, and supported by Touch Medical Media.

Review Process: Double-blind peer review.

Compliance with Ethics: This article is an opinion piece and does not report on new clinical data, or any studies with human or animal subjects performed by any of the authors.

Authorship: All named authors meet the criteria of the International Committee of Medical Journal Editors for authorship for this manuscript, take responsibility for the integrity of the work as a whole and have given final approval for the version to be published.

Received: August 7, 2019

Accepted: October 9, 2019

Citation: US Endocrinology. 2019;15(2):65-7

Corresponding Author: John Doupis

Diabetes Division and Clinical Research Center, latriko Paleou Falirou Medical Center,

36 Areos Street, Paleo Faliro, 17562, Athens, Greece.

E: john.doupis@joslin.harvard.edu

Support: No funding was received in the publication of this article.
Cardiovascular outcome trials (CVOTs) have become mandatory for glucose-lowering drugs in the treatment of patients with type 2 diabetes (T2D) since 2008, following the withdrawal of rosiglitazone from the market because of its association with increased risk of cardiovascular disease (CVD). ${ }^{1}$ In subsequent years, results have emerged from several CVOTs, with many more underway, all demonstrating the noninferiority of glucose-lowering agents to placebo in terms of CV outcomes. ${ }^{2}$ In 2015, the EMPA-REG trial demonstrated that the sodium-glucose cotransporter-2 (SGLT2) inhibitor empagliflozin (JARDIANCE ${ }^{\circledR}$, Boehringer Ingelheim, Ridgefield, CT, USA) significantly reduced the rate of $\mathrm{CV}$ events compared with placebo. ${ }^{3}$ Since then, other studies, including CANVAS with the SGLT inhibitor canagliflozin (INVOKANA ${ }^{\oplus}$, Janssen, Beerse, Belgium); ${ }_{4}^{4}$ LEADER, with the injectable once-daily glucagon-like peptide 1 (GLP-1) agonist, liraglutide (Victoza ${ }^{\circledR}$, Novo Nordisk, Bagsværd, Denmark), ${ }^{5}$ SUSTAIN-6 with semaglutide (Ozempic ${ }^{\circledast}$, Novo Nordisk, Bagsværd, Denmark); and DECLARE-TIMI 58 with the SGLT inhibitor, dapaagliflozin (FARXIGA ${ }^{\oplus}$, AstraZeneca, Cambridge, UK) ${ }^{7}$ and have also shown clinically meaningful CV benefits.

However, CVOTs to date have a number of important limitations. They all involved patients either with established CVD or chronic renal failure at baseline, or at high risk of CVD. In addition, a subgroup analysis of the LEADER trial suggested that liraglutide effectively lowered CV risk only in people with established CVD. ${ }^{5}$ It is, therefore, difficult to extrapolate the study findings to the entire population of people with T2D. In addition, CVOTs to date have only addressed short-term outcomes, although many complications of T2D-including blindness, renal failure, amputation, CV death-do not usually appear within the first 5 years. ${ }^{8}$ There is a need for CVOTs involving lower-risk patients who have not yet developed CVD to determine whether their risk of developing CVD can be reduced. Such studies would need a large sample size and long follow-up to achieve a statistically significant number of major adverse CV events (MACE) events, but would provide valuable information about CVD prevention.

The most recent CVOT, the REWIND study (ClinicalTrials.gov identifier: NCT01394952), evaluated the CV safety of the once-weekly injectable GLP-1 receptor agonist dulaglutide (Trulicity ${ }^{\circledR}$, Lilly, Indianapolis, IN, USA). ${ }^{9}$ This is the first CVOT with a GLP-1 receptor agonist to include a majority of patients who did not have established CVD (defined as prior myocardial infarction [MI], ischemic stroke, unstable angina, revascularization, hospitalization for ischemia-related events, or documented myocardial ischemia) at baseline - only 31\% of the participants had established CVD. Compared to other CVOTs, this better reflects the broader T2D patient population seen in routine clinical practice. However, participants were still at high risk of CV events: the study included patients aged $\geq 50$ with established CVD, and patients $\geq 60$ years old with risk factors. The 9,901 participants from 371 sites across 24 countries were aged $\geq 50$ years, had a mean duration of T2D of 10 years and a mean baseline glycated 
Figure 1: REWIND trial—primary endpoint of major cardiovascular events

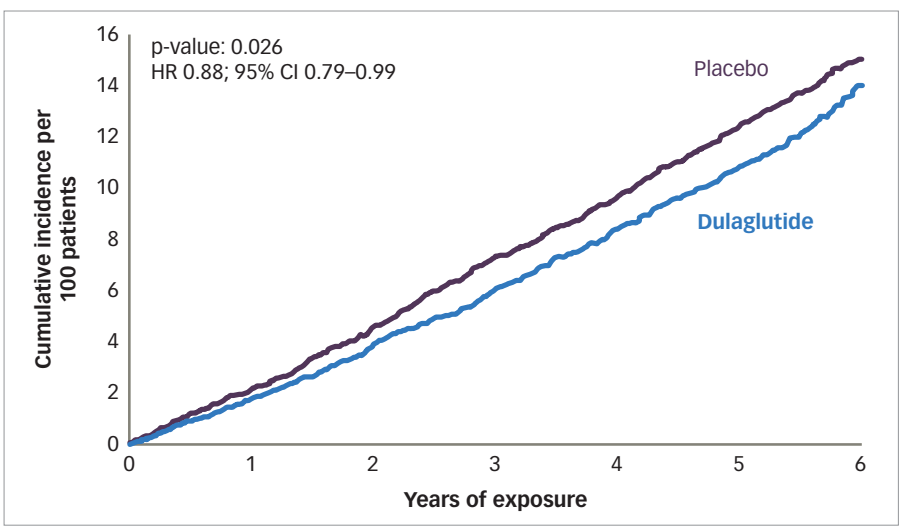

Major cardiovascular events included cardiovascular death, non-fatal myocardial infarction, or non-fatal stroke.

$\mathrm{Cl}=$ confidence interval; $\mathrm{HR}=$ hazard ratio

Reused with permission from The Lancet. Source: Gerstein et al. 2019.10

Figure 2: Individual cardiovascular outcomes of the REWIND trial

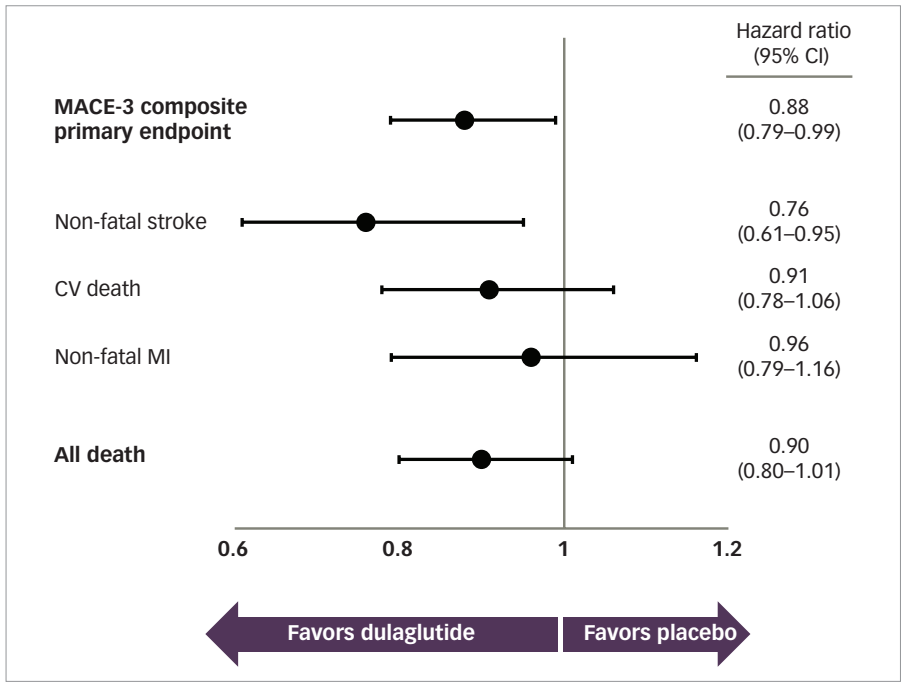

$\mathrm{Cl}=$ confidence interval; $\mathrm{CV}=$ cardiovascular $; \mathrm{MACE}=$ major cardiovascular events $\mathrm{MI}=$ myocardial infarction

Reused with permission from The Lancet. Source: Gerstein et al. 2019.10

hemoglobin ( $\mathrm{HbA} 1 \mathrm{C})$ of $7.2 \%$-lower than has been seen in previous studies. However, this is not the largest CVOT to date; the EXSCEL study enrolled over 14,000 participants ${ }^{10}$ and the DECLARE-TIMI 58 study enrolled over 17,000 participants. ${ }^{7}$ Patients were randomly assigned (1:1) to either weekly subcutaneous injection of dulaglutide $(1.5 \mathrm{mg})$ or placebo. ${ }^{9}$

REWIND had a median follow-up of 5.4 years, the longest to date for a CVOT for a GLP-1 receptor agonist. By comparison, the DECLARE study continued for 4.2 years. ${ }^{7}$ The outcome of first occurrence of MACE (including nonfatal MI, non-fatal stroke, or death from CV causes) occurred in $12.0 \%$ of dulaglutide-treated patients, an incidence rate of 2.4 per 100 person-years compared with $13.4 \%$ in the placebo group, an incidence rate of 2.7 per 100 person-years (hazard ratio [HR] 0.88; 95\% confidence interval [Cl] 0.79-0.99; $\mathrm{p}=0.026$ ) (Figure 1). The difference in MACE rates between the two study
Table 1: Stroke incidence in cardiovascular outcome trials

\begin{tabular}{|c|c|c|c|}
\hline Study name & Drug name & $\begin{array}{l}\text { Hazard ratio for } \\
\text { stroke }\end{array}$ & $p$-value \\
\hline EMPA-REG ${ }^{13}$ & Empagliflozin & 1.18 & 0.26 \\
\hline CANVAS $^{4}$ & Canagliflozin & 0.87 & Not stated \\
\hline LEADER $^{5}$ & Liraglutide & 0.86 & 0.16 \\
\hline SUSTAIN-66 & Semaglutide & $0.61 *$ & 0.04 \\
\hline DECLARE-TIMI $58^{7}$ & Dapagliflozin & 1.01 & Not stated \\
\hline EXSCEL $^{9}$ & Exenatide & 0.85 & Not stated \\
\hline REWIND ${ }^{10}$ & Dulaglutide & 0.76 & 0.017 \\
\hline
\end{tabular}

* Data only available for nonfatal stroke. ns=non-significant.

arms could be seen by 1 year of treatment, and increased proportionally over the following years. ${ }^{9}$

In terms of secondary outcomes, there were no significant long-term benefits for dulaglutide against nonfatal $\mathrm{Ml}$ (HR 0.96; 95\% Cl 0.79-1.16; $\mathrm{p}=0.65$ ), and minimal benefit against $\mathrm{CV}$ death (HR 0.91; 95\% $\mathrm{Cl} 0.78-1.06$; $\mathrm{p}=0.21$ ), which is something quite reasonable and expected, as the majority of the study subjects at baseline, were presenting relatively low morbidity. However, there was a substantial and significant benefit against nonfatal stroke (HR 0.76; 95\% Cl 0.6-0.95; $\mathrm{p}=0.017$ ) (Figure 2). By comparison, other recent CVOTS have not shown substantial reductions in stroke: HR 1.01 in DECLARE-TIMI 58.7 Patients receiving dulaglutide benefit had a $10 \%$ reduced incidence of all-cause death over 5 years compared with those receiving placebo (HR 0.90; 95\% Cl 0.80-1.01). Subgroup analysis revealed that dulaglutide was most beneficial for patients aged $<66$ years $(10.0 \%$ of the study population; rate per 100 years: 1.9$)$, females (9.5\%; rate per 100 years: 1.8), and patients with T2D of $<5$ years' duration (10.4\%; rate per 100 years: 2.0), although $p$ values were not adjusted for multiple testing, and were not significant.

In addition, the REWIND study provided additional evidence for dulaglutide's efficacy in treating T2D, providing modest changes in $\mathrm{HbA} 1 \mathrm{C}(-0.46 \%$ versus $+0.16 \%$ with placebo) and weight (-2.95 kg versus $-1.49 \mathrm{~kg}$ with placebo). ${ }^{9}$ Renal outcomes were secondary endpoints of REWIND and comprised a composite microvascular outcome, defined as the first occurrence of new macroalbuminuria (urinary albumin-to-creatinine ratio $>33.9 \mathrm{mg} / \mathrm{mmol}$ ), a sustained decline in estimated glomerular filtration rate of $\geq 30 \%$ from baseline, or chronic renal replacement therapy. This composite outcome occurred in $17.1 \%$ of participants in the dulaglutide group and $19.6 \%$ of those in the placebo group (HR 0.85; 95\% Cl 0.77-0.93; $\mathrm{p}=0.0004$ ). ${ }^{9}$

The safety profile of dulaglutide was consistent with that of other GLP-1 receptor agonists. Dulaglutide was well-tolerated among the study population. There was higher rate of gastrointestinal adverse events (47.4\%) among patients taking dulaglutide than those taking placebo (34.1\%; $p<0.0001)$.

In an accompanying editorial, Subodh Verma, David Mazer, and Vlado Perkovic, all from the University of Toronto, Ontario, Canada, commented that the magnitude of benefit in terms of the composite CV outcome (12\%) was modest and numerically lower than that seen in the other GLP-1 agonist studies i.e., LEADER, SUSTAIN-6, and HARMONY (it should be mentioned that SUSTAIN was designed as a non-inferiority trial). However, the outcome 
was consistent with the overall effect size from a meta-analysis of all previous GLP-1 receptor agonist trials. Additionally, they highlighted the fact that participants in the REWIND trial were at lower risk of CV events than participants in the previous studies. ${ }^{11}$

The stroke reduction seen with dulaglutide is not a typical finding of CVOTs. Interestingly, the only other CVOT, to date, to show a substantial and significant reduction of stroke was SUSTAIN-6, with the GLP-1 receptor agonist semaglutide (Table 1). ${ }^{6} \mathrm{~A}$ recent systematic review and meta-analysis found that GLP-1 receptor agonists showed a significant reduction, by $13 \%$, in the risk of total stroke, suggesting possible neuroprotective effects. ${ }^{12}$
The fact that the primary outcome of the REWIND study seemed to be driven largely by a reduction in stroke rather than a reduction in MI may need further investigation. Nevertheless, these are valuable clinical findings. This international study's broad patient population, including a high proportion of women (46\%), people without established CVD and inclusion of participants with a lower mean baseline HbA1c (7.2\%) suggest that the findings will be directly applicable to patients with T2D seen in everyday clinical practice worldwide. The study authors concluded that dulaglutide could be considered for the management of glycemic control in middle-aged and older people with T2D with either previous CVD or CV risk factors. ${ }^{9}$
1. Singh $\mathrm{S}$, Loke YK, Furberg CD. Long-term risk of cardiovascular events with rosiglitazone: a meta-analysis. JAMA 2007;298:1189-95.

2. Cefalu WT, Kaul S, Gerstein HC, et al. Cardiovascular outcomes trials in type 2 diabetes: where do we go from here? Reflections from a diabetes care editors' expert forum. Diabetes Care. 2018;41:14-31.

3. Zinman B, Wanner C, Lachin JM, et al. Empagliflozin cardiovascular outcomes, and mortality in type 2 diabetes. N Eng/ J Med. 2015;373:2117-28.

4. Neal B, Perkovic V, Mahaffey KW, et al. Canagliflozin and cardiovascular and renal events in type 2 diabetes. N Eng/ J Med. 2017;377:644-57.

5. Marso SP, Daniels GH, Brown-Frandsen K, et al. Liraglutide and cardiovascular outcomes in type 2 diabetes. N Engl I Med. 2016;375:311-22.

6. Marso SP, Bain SC, Consoli A, et al. Semaglutide and cardiovascular outcomes in patients with type 2 diabetes. N Eng/ J Med. 2016;375:1834-44.

7. Wiviott SD, Raz I, Bonaca MP, et al. Dapagliflozin and cardiovascular outcomes in Type 2 diabetes. N Engl J Med. 2019;380:347-57.

8. Holman RR, Paul SK, Bethel MA, et al. 10-year follow-up of intensive glucose control in type 2 diabetes. N Eng/ J Med. 2008;359:1577-89.

9. Gerstein $\mathrm{HC}$, Colhoun, HM, Dagenais, GR, et al. Dulaglutide and cardiovascular outcomes in type 2 diabetes (REWIND): a double-blind, randomised placebocontrolled trial. Lancet. 2019;394:121-30.

10. Holman RR, Bethel MA, Mentz RJ, et al. Effects of once-weekly exenatide on cardiovascular outcomes in type 2 diabetes. N Eng/ J Med. 2017;377:1228-39.

11. Verma $S$, Mazer $C D$, Perkovic V. Is it time to REWIND the cardiorenal clock in diabetes? Lancet. 2019;394:95-7.

12. Barkas F, Elisaf M, Milionis H. Protection against stroke with glucagon-like peptide 1 receptor agonists: a systematic review and meta-analysis. Eur J Neurol. 2019;26:559-65.

13. Zinman B, Wanner C, Lachin JM, et al. Empagliflozin, cardiovascular outcomes, and mortality in type 2 diabetes. N Eng/ J Med. 2015;373:2117-28. 